\title{
Pavement raveling detection and measurement from synchronized intensity and range images
}

\author{
S Mathavan \\ Visiting Research Fellow \\ School of Architecture, Design and the Built Environment, \\ Nottingham Trent University \\ Burton Street, Nottingham NG1 4BU, UK \\ E-mail: s.mathavan@ieee.org
}

\author{
M M Rahman \\ Senior Lecturer \\ Department of Civil Engineering \\ Brunel University \\ Kingston Lane, Uxbridge, Middlesex UB8 3PH \\ tel: +44 (0)1895 267590 \\ mujib.rahman@ntu.ac.uk \\ M Stonecliffe-Jones \\ Head of European Consultancy \\ Dynatest UK Limited \\ Unit 12 Acorn Enterprise Centre, \\ Frederick Road, Kidderminster, DY11 7RA, UK \\ mstonecliffe-jones@dynatest.com \\ K Kamal \\ Assistant Professor \\ Department of Mechatronics Engineering, \\ College of Electrical and Mechanical Engineering, \\ National University of Science and Technology, \\ Rawalpindi, Pakistan \\ khurramkkamal@hotmail.com
}

\begin{abstract}
Raveling on asphalt surfaces is a loss of fine and coarse aggregates from the asphalt matrix. The severity of raveling could be an important indicator of the state of pavements as excessive raveling not only reduces the ride quality, but eventually leads to pothole formation or cracking. Hence, it is importatnt to detect and quantify raveling. In this paper, an effort has been made, for the first time, to quantify raveling from a combination of 2D and 3D images. First, a texture descriptor method called Laws' texture energy measure is used in conjunction with the Gabor filter and other imorphological operation to distinguish road areas from others. Then, signal processing techniques are used to detect and quantify raveling. Hundreds of industrial images are used to test as well as to show the promise of the proposed algorithm.
\end{abstract}

Key word: Raveling, range images, 3D imaging, Gabor filter, Laws’ texture energy, region segmentation

Corresponding author

Submission date: 31/07/2013

Word count: 4932, 11 Figures 


\section{INTRODUCTION}

It is fundamental for road authorities across the world, to define, firstly, a data collection method to acquire knowledge of the pavement condition within a limited time and management cost, without traffic disruption, ensuring safety of the workforce and the traffic in general. In the last twenty years, the rapid advancement in the processing power of computer, communication, laser and imaging technology made it possible to collect and analyze large amount of road surface distress information, avoiding high degree of variability, providing meaningful quantitative information, and leading to avoid inconsistencies. Current methods for distress identification use equipped vehicles with high resolution cameras and sensors to record pavement surface images and profile at traffic speed providing accurate information for optimal maintenance and rehabilitation needs despite some limitation still exist on the accuracy of crack detection [1]. Another major issue with the 2D video-based systems is their inability to discriminate dark areas not caused by pavement distress such as tire marks, oil spills, shadows, and recent filings [2]. Moreover, the shadows and poor illumination are also major problems for daytime operation though they can overcome using additional lighting systems or by acquiring data in the night after sunset [3].

Other than the more traditional 2D image analysis to detect pavement distress (cracks, patches, potholes, etc.), new systems and procedures are proposed to obtain 3D pavement evaluation which has the potential to capture more accurate surface features and extract and quantify information that were extremely difficult from the 2D dimensional survey. For example, until now, the extent and the severity measurement of raveling has been a subjective assessment, estimating the area with the missing stone.

\section{APPLICATION OF 3D TECHNOLOGY IN PAVEMENT CONDITION SURVEY}

Research in 3D technology for pavement evaluation is a recent development. Therefore, the literature in this area is limited. The 3D laser, photogrammetry and stereo vision techniques are the most popular among various types of 3D technology available [4,5]. All these systems have great potentials but also have limitations when equipment and management costs are considered. There are two main challenges to overcome before they are widely used in pavement evaluation. The first one is to capture the image in a consistent manner overcoming the effect of lighting, shadows, etc. The second challenge is to develop a fast and accurate algorithm to separate different defects accurately. The following sections highlight the key research on the 3D image capturing and processing techniques.

An early systems for the 3D imaging of pavement surfaces was based on the photogrammetric principle [6]. Although the system yielded good results, ensuring lighting requirement was very difficult for the paired camera used in the system to obtain high fidelity 2D images of the pavement surfaces. Another system, known as LIDAR (Light Detection and Ranging) was widely used which composed of a rotating laser scanning system, GPS receiver and an IMU [7]. Although initially the system attracted widespread attention, due to the difficulty in making significant improvement in the resolution of the system in the last decades, and the popularity of laser based 2D imaging system, the usage of this technique has been limited to niche applications [7].

In 2008, Laurent et al proposed a 3D Transverse Laser Profiling System for the Automatic Measurement of Road Cracks, which then subsequently implemented as a commercial system with a custom made software to preprocess the data [8]. In this system, known as Laser Crack Measurement System (LCMS), high-speed cameras were used together with custom optics and laser line projectors to acquire both 2D images and highresolution 3D profiles of the road. The system could be operated by night or by day under all types of lighting conditions - in both sunlit and shaded areas. Various pavement types like regular or open-graded asphalt, chipseal and concrete, can be measured at survey speeds up to $100 \mathrm{~km} / \mathrm{h}$, and on roads reaching $4 \mathrm{~m}$ in width.

Wang et al also used the same technique as LCMS, developed a prototype automated vehicular platform including laser based sensors that can capture 1mm resolution 3D representation of pavement surface even in adverse lighting condition and the development of an algorithm and software to produce results on pavement distresses [9]. However, the software, Pavevision 3D, used in this system has substantially better performance than the LCMS in terms of 3D line rate and, 2D visual data. Other recent laser based 3D system is being proposed by [10], where a real-time 3D scanning system was used for the inspection of pavement distortion such as visualization of rutting and shoving using a high-speed 3D transverse scanning techniques based on structured light triangulation. To improve the accuracy of the system, a multi-view coplanar scheme was employed in the calibration procedure so that more feature points can be used and distributed across the field of view of the camera. A sub-pixel line extraction method is applied to the laser stripe location, which includes filtering, edge detection and spline interpolation. The pavement transverse profile is then generated from the laser stripe curve and approximated by line segments. Sun et al proposed a new method of analysis based on the 
sparse representation to decompose the pavement profile signal into a summation of the mainly pavement profile and cracks [11].

\section{RAVELING OF ASPHALT SURFACES}

In simple terms, raveling on asphalt surfaces is a loss of fine and coarse aggregates from the asphalt matrix due to the adhesion failure at the interface. In many cases raveling is a combination of more than one contributing physical mechanisms by which the aggregate is separated from the binder; such as surface type, improper mixture design (lower binder content then the specification, high proportion of dust), inadequate compaction, weathering, traffic, ageing of bitumen, the high intense hydrostatic pressure created by a combination of traffic and water entering the pavement through interconnecting voids [12, 13], moisture or freeze-thaw cycles due to seasonal variation, effect of snow plowing in winter months. Excessive raveling not only reduces the ride quality, but eventually leads to pothole formation or cracking. In addition, in recent years surface dressing and other thin surfacing systems are increasingly used as a means of preventative maintenance for pavement preservation. These surfaces are prone to reveling because of combination of factors as mentioned earlier. Therefore, there the severity of raveling could be an important informant to evaluate the state of the pavement.

The measurement of raveling is based on the visual observation rather than any derived quantification. The severity level is rated by the degree of aggregate loss within a segment of a road. The segment is typically one tenth of the mile or a kilometer and expressed relative to the surface area of the surveyed lane. It is important to note that raveling is measured or observed differently depending on the surface type. For Bituminous Surface Treatment (BST) raveling is caused by the loss of aggregate and the binder is exposed. On the other hand, for chip sealed pavements, as they tend to look raveled because of the inherent nature of the chip seal surface, it may be mistaken as raveling which is actually an excess asphalt resulting loss of aggregate, and should be rated as flushing [12]. The various stages of raveling are usually described as light (loss of surface fines), moderate (loss of fines and some larger aggregate exposed), and severe (loss of fine and coarse aggregate). The extent of raveling could be localized (patchy areas, usually in the wheel paths), on the wheel path (majority of wheel tracks is affected, but little or none elsewhere in the lane) or could extend through the entire lane width (most of the lane is affected) [12].

\section{RESEARCH OBJECTIVES}

In this paper Laws' texture energy measures are used to detect texture boundaries in intensity (i.e. 2D) images (Figure 1) to distinguish road surfaces from lane marking and other painted surfaces. In addition, the Gabor filter, a frequency domain based technique, is used to enhance the edges that result from the texture boundary detection as described above. Furthermore, some morphological operations are performed to further improve the segmentation accuracy.
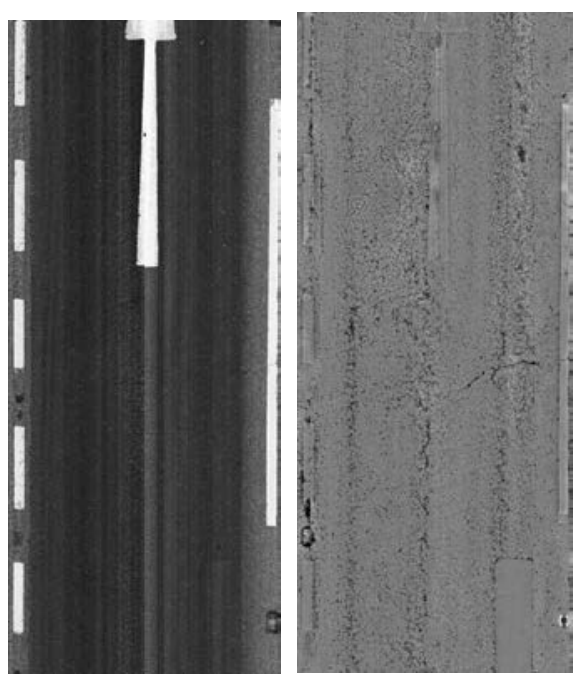

FIGURE 1 An intensity image and its corresponding range image [Courtesy Dynatest UK Ltd.] 


\section{LAWS’ TEXTURE ENERGY MEASURES}

Surfaces can be distinguished from their texture. Several texture analysis methods exist. Co-occurrence matrices, autocorrelation features and wavelet-based methods are to name a few [14]. The laws' texture energy method measures the amount of texture variation within a finite-sized window. Texture energy is computed within a 5x5 window, usually. A number of masks are formed from the vectors shown below.

$$
\begin{array}{ll}
\text { L5 (Level) } & =\left[\begin{array}{rrrrr}
1 & 4 & 6 & 4 & 1
\end{array}\right] \\
\text { E5 (Edge) } & =\left[\begin{array}{rrrrr}
-1 & -2 & 0 & 2 & 1
\end{array}\right] \\
\text { S5 (Spot) } & =\left[\begin{array}{rrrrr}
-1 & 0 & 2 & 0 & -1
\end{array}\right] \\
\text { R5(Ripple) } & =\left[\begin{array}{rrrrr}
1 & -4 & 6 & -4 & 1
\end{array}\right]
\end{array}
$$

$\mathrm{R} 5$ vector has been designed to detect ripples in the images. The rationale behind these detections is that the texture of a given image can be broken down into very fundamental geometric shape descriptions like edges, spots, levels, etc. These 4 vectors are then used to form $5 \times 5$ masks.

The mask L5S5 is formed in the following manner: $\left[\begin{array}{l}1 \\ 4 \\ 6 \\ 4 \\ 1\end{array}\right] \times\left[\begin{array}{lllll}-1 & 0 & 2 & 0 & -1\end{array}\right]=\left[\begin{array}{ccccc}-1 & 0 & 2 & 0 & -1 \\ -4 & 0 & 8 & 0 & -4 \\ -6 & 0 & 12 & 0 & -6 \\ -4 & 0 & 8 & 0 & -4 \\ -1 & 0 & 2 & 0 & -1\end{array}\right]$

In total, 16 such masks are formed: L5L5, L5E5, L5S5, L5R5, E5L5, E5E5, E5S5, E5R5, S5L5, S5E5, S5S5, S5R5, R5L5, R5E5, R5S5 and R5R5.

Pavement images are convolved with the above masks as shown in Figure 2.

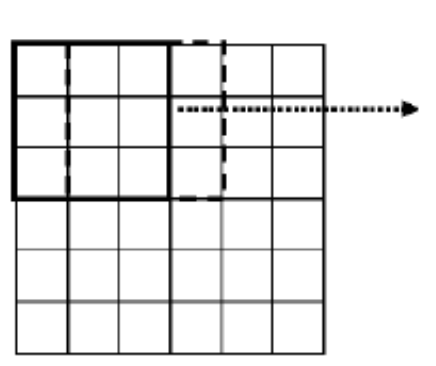

Input image

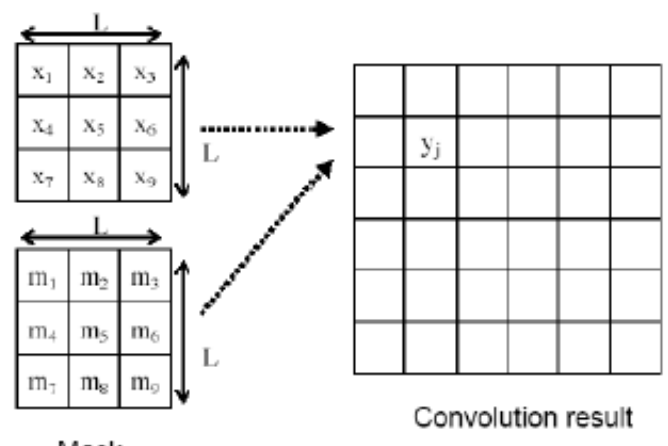

Mask
Convolution result

FIGURE 2 Mask convolution. $y_{i}=\sum_{i=1}^{N} x_{i} \times m_{i}$, where Pixels $=N=L \times L$ [15]

The resulting image from convolution can be used to detect texture boundaries.

\section{GABOR FILTER}

The Gabor filter is a frequency based technique that has been used for object recognition, edge detection and optical character recognition. This filter is very special in the sense that visual cortex cells in mammals can be expressed by Gabor functions. The filter has the ability to respond to different orientations, hence it helps distinguish objects oriented in different directions. The Gabor filter is implemented as a filter bank consisting of filters with a number of orientations (see $\theta$ below).

The Gabor filter is formed by the modulation of a Gaussian envelope by a complex sinusoid. The filter's real part can be expressed as follows,

$g_{i}(x, y)=\frac{1}{2 \pi \sigma^{2}} e^{-\frac{1}{2 \sigma^{2}}\left[\left(x^{\prime}\right)^{2}+\left(\gamma y^{\prime}\right)^{2}\right]}\left\{\sin \left[\frac{2 \pi}{\lambda}(x \cos \theta+y \sin \theta)+\psi\right]\right\}$

Where, $x^{\prime}=x \cos \theta+y \sin \theta$ and $y^{\prime}=-x \sin \theta+y \cos \theta$. Here $\sigma$ is the standard deviation of the Gaussian neighborhood in the $x^{\prime}$ direction. $\gamma$ is the ellipticity of the filer. $\theta$ is desired orientation of the filter, $\lambda$ is the wavelength of the sinusoid, $\psi$ is the phase offset of the modulation factor, which decides the symmetry or anti- 
symmetry of the filter and the width $(a)$ and the length $(b)$ of the elliptical Gaussian (2D) envelope and the angle between the orientation of the sinusoidal wave vector and the two dimensional Gaussian axes.

Figure 3 shows three different Gabor filters where the orientation, $\theta$, or the wavelength, $\lambda$, is changed. The pictures depict the continuous domain representations of the Gabor filter.
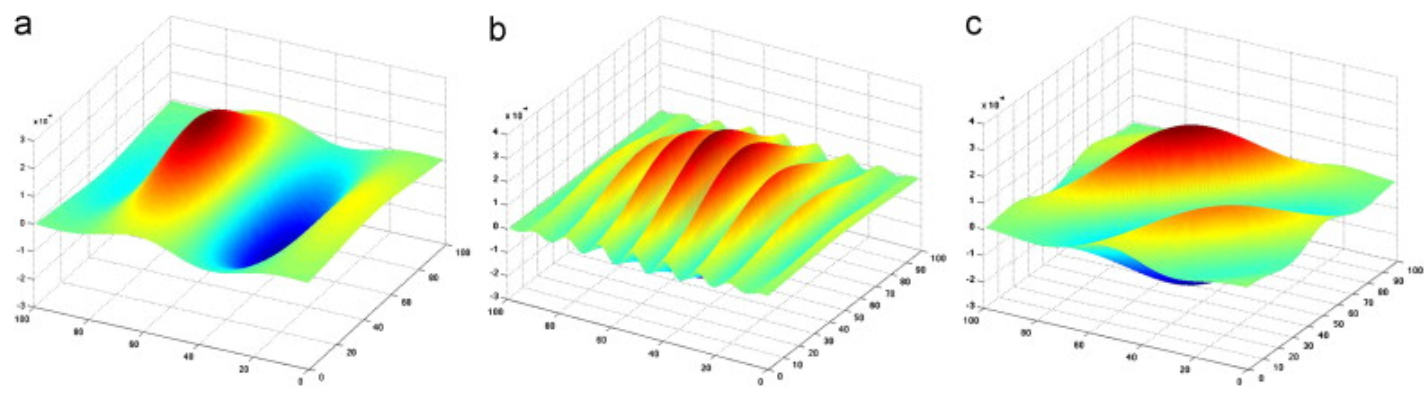

FIGURE 3 Gabor filter with $\theta=0$, $\lambda=2$ (a), Gabor Filter with $\theta=0, \lambda=0.5$ (b) and Gabor filter with $\theta=\pi / 4, \lambda=5$ (c) [16]

For practical image processing applications the continuous function has to be digitized and represented by a mask as discussed in the previous section. Then the image is convolved with the mask as explained in Figure 2. For a detailed explanation of the theory, refer to [17], where the Gabor filter is utilized for pavement crack detection.

\section{MORPHOLOGICAL OPERATIONS ON BINARY IMAGES}

Binary images are the images that consist only black (gray level 0) and white pixels (gray level 1), i.e. the image has two intensity levels only. Blobs in binary images are the collection of white pixels that are connected by a neighborhood. A lone white pixel, that does not have any neighbors, is also considered as a blob. According to 8 neighborhoods, the middle pixel with value $x_{5}$ in Figure 2 will have all the 8 pixels surrounding it $\left\{x_{1}, x_{2}, x_{3}, x_{4}\right.$, $\left.x_{6}, x_{7}, x_{8}, x_{9}\right\}$ as its neighbors. When it comes to 4-neighborhood, the pixels $\left\{x_{2}, x_{4}, x_{6}, x_{8}\right\}$ are considered the neighbors of the middle pixel, $x_{5}$. The corner connectivity is not considered for a 4-neighborhood. In this paper only 8 neighborhood is used (this is the default option with MATLAB's Image Processing Toolbox's morphological functions).

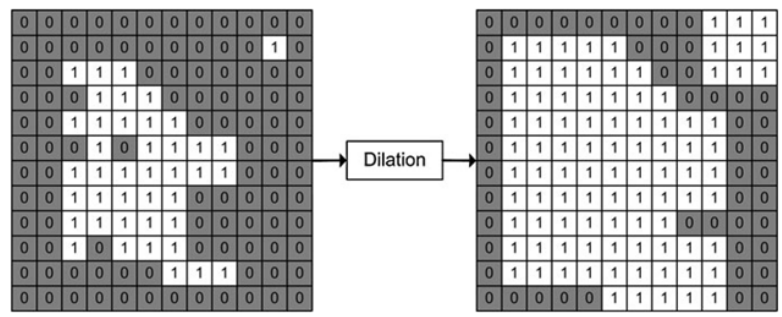

(a)

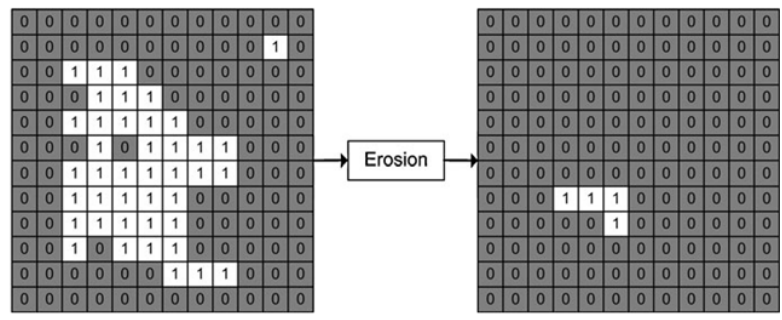

(b)

FIGURE 4 Two morphological operations: dilation (a) and erosion (b) performed with 8 pixel connectivity on an image with two blobs [18]

A number of morphological operators are available. Out of these, dilation and erosion are used in this project. Image morphology is frequently used for image enhancement. In a general sense, dilation adds white pixels to an image based on any given criteria. Whereas, the erosion operation removes white pixels from the image. 
Any black pixel that has a white pixel in its 8 - neighborhood is turned into a white pixel (i.e. its value is set to 1). This operation is depicted in Figure 4 (a), where the image on the left is the original image and the right one shows the dilated version. To explain it more, for the lone white pixel in the top right corner of the original image in Figure 4(a), the dilation operation makes all the pixels in its 8-neighborhood white. The bwmorph function of MATLAB has been used with the dilation option chosen [19]

\section{Erosion}

Erosion removes any white pixels that have at least one black pixel in its 8-neighborhood (See Figure 4 (b)). Only the four white pixels, shown in the image after erosion, have white pixels entirely in their 8-neighborhood . Once again MATLAB's bwmorph is employed used with the dilation option [19].

Two other operations are also performed on the images. Boundary extraction, extracts the boundaries of every blob in the image based on 8-neighborhood. MATLAB function boundaries are used to extract blob boundaries. Hole filling replaces the black pixels, fully inside a blob, with white ones. For this purpose imfill function of MATLAB is employed.

\section{EXPERIMENTATION}

\section{Image Acquisition}

The 2D and 3D images used in this study are obtained by Dynatest UK Ltd. using the LCMS system on a road section in the UK. There is some post-processing on the images by the Pavemetrics software and the resulting are 2D (i.e. intensity) and 3D (i.e. Range) both of which are 8-bit grayscale images. The size of the road imaged by the systems is $10 \times 4.16 \mathrm{~m}^{2}$. Both the images have a resolution of $2500 \times 1040$. Hence, once pixel images an area of $4 \times 4 \mathrm{~mm}^{2}$ on the road. Ninenhundred 2D-3D image pairs have been supplied by Dynatest.

\section{Texture Edge Detection: Laws' texture energy masks}

The target here is to segment road areas from the paints, lines, etc. found in the images and then to look for raveled areas, preferably using the range images.. The use of 3D image for the purpose of region segmentation is very limited as distinguishing different surfaces from each other using $3 \mathrm{D}$ imaging can result in ambiguity. This is especially the case for a road surface, as it can be smooth and rough at two different locations at ground level. This variation will result in 3D images with low and high range fluctuations. Furthermore two different surfaces can exhibit same smoothness, in their range values, as such cannot be differentiated. Hence, range images are not very good for distinguishing different surfaces. Intensity (i.e. 2D) images are used to extract road surface in this study.

As seen in Figure 5 (a), the intensity images are complex with varying amounts of image intensities, within any given type of surface. The texture within a given surface change greatly as well. Due to these variations, image segmentation techniques like thresholding and edge detection are not found to give effective results. To segment different the road regions from the non-road areas, the 16 masks based on Laws' texture energy measured are used to convolve the $2 \mathrm{D}$ intensity image.

It is experimentally found that the average of the images resulting from the convolution with the masks S5L5 and L5S5 give the best texture segmentation for these images. The corresponding texture edge image is shown in Figure 5 (b). It can be seen from Figure 5 (b) that many of the pseudo edges, which are due to intensity variations, are eliminated, esp. when compared with Figure 5 (a). However, many micro edges are still detected, preventing a clear segmentation between road and other regions. To eliminate these a thresholding operation is performed. The thresholded, hence binary, image is shown in Figure 5 (c). The segmentation is not perfect as there remain lots of edges within the letters ' $S$ ', ' $L$ ' and ' $W$ ', written on the road. In addition, the lane markings that are at the right- and leftmost regions of the image, have line segments missing. 


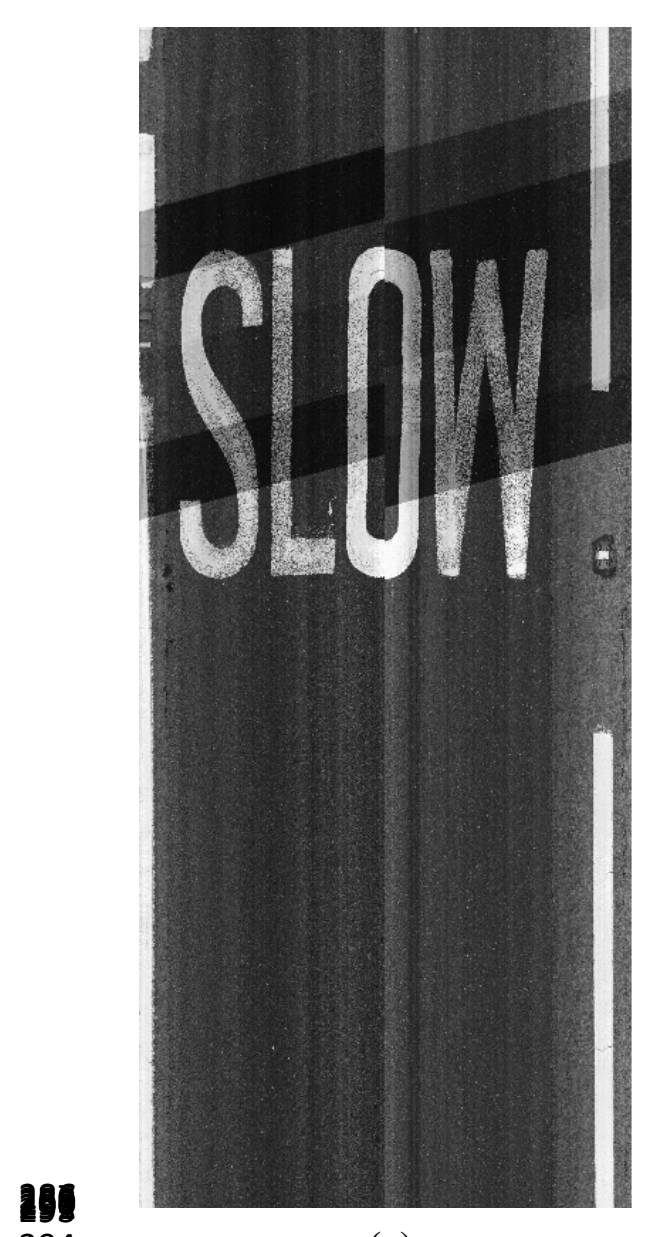

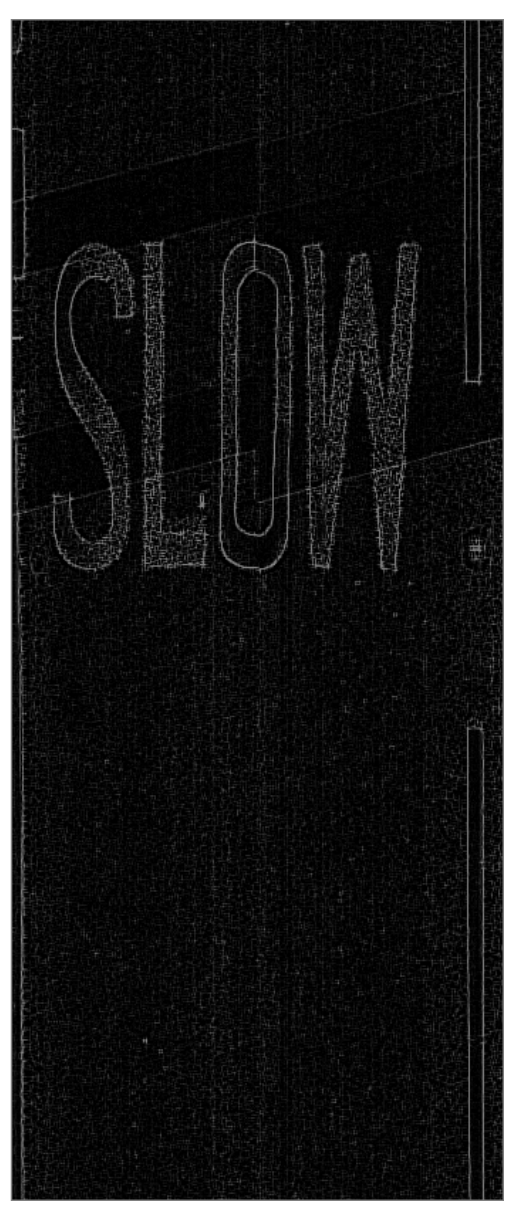

(b)

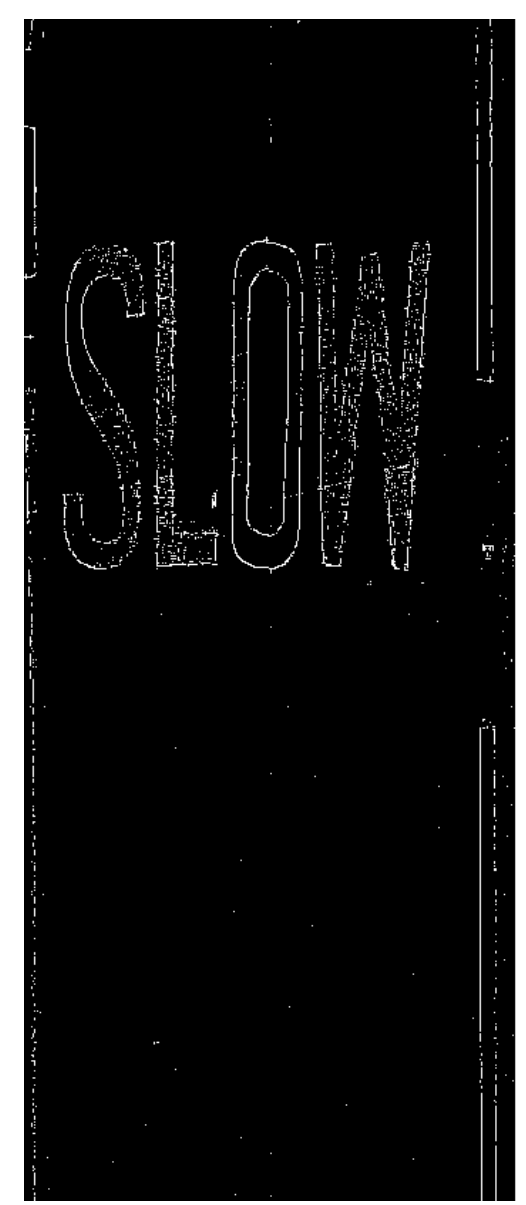

(c)

FIGURE 5 An intensity image (a), its Laws' texture edge image (b) and the threshold edge image (c)

\section{Texture Edge Enhancement: The Gabor Filter}

Twelve Gabor filters at orientations $\left\{0^{\circ}, 15^{\circ}, 30^{\circ}, 45^{\circ}, 60^{\circ}, 75^{\circ}, 90^{\circ}, 105^{\circ}, 120^{\circ}, 135^{\circ}, 150^{\circ}, 165^{\circ}\right\}$ are used to convolve the image given in 5 (c). Other parameters used for the Gabor filters are, $\gamma=1, \sigma=12, \lambda=40$ and $\psi=0$. All 12 images resulting from the filters are then thersholded. Figures 6 (a) and 6 (b) show the threshold images for the filter orientations, $\theta$, of $0^{\circ}$ and $90^{\circ}$, respectively.

As seen in Figure 6 (a), the orientation $\theta=0^{\circ}$ picks up all the horizontal edges in Figure 5 (c). From Figure 6 (b), it can be seen that the $90^{\circ}$ orientation Gabor filter detects all the vertical edges. In addition to orientation based edge detection, Gabor filters also have a smearing effect hence fill up the gaps (non-detections) in the line segments in Figure 5 (c). The main disadvantage is that the line segments tend to get thicker after processing with Gabor filters. However, this drawback works to the advantage of this project, as it leads to a more conservative detection, i.e. under detection, of the road surface. 


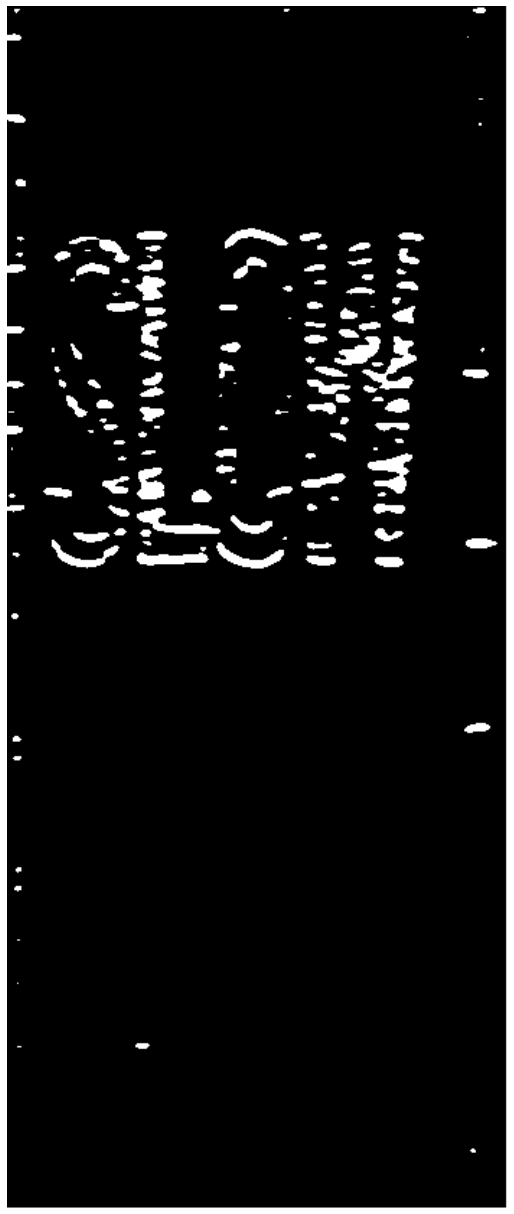

(a)

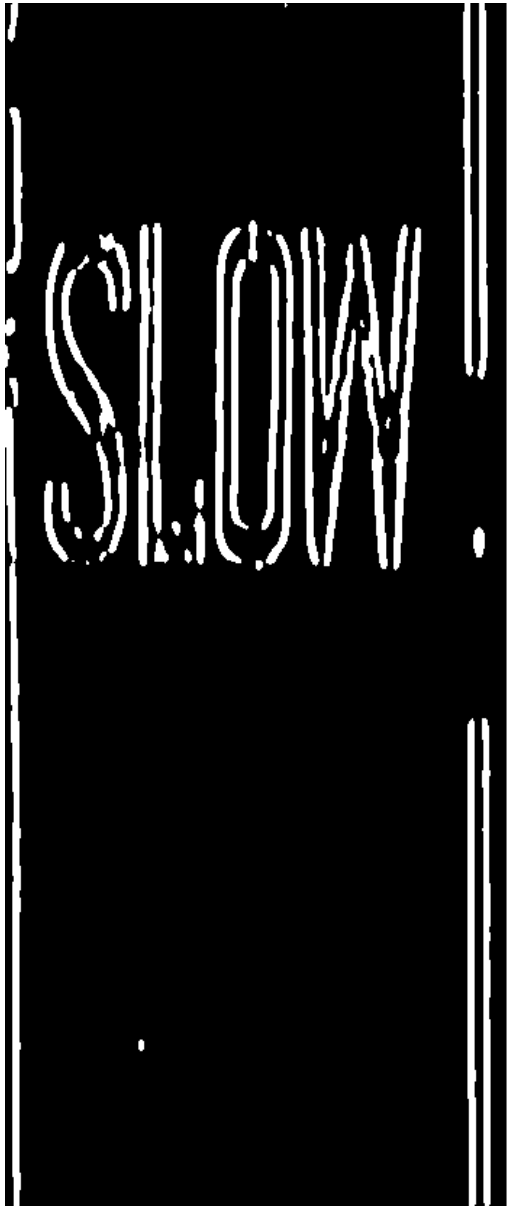

(b)

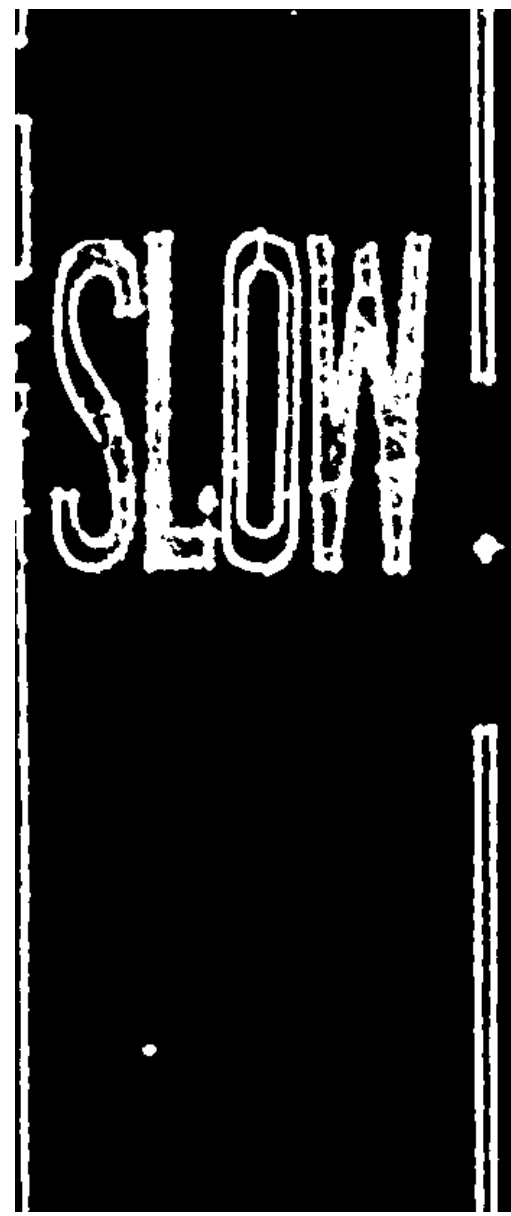

(c)

FIGURE 6 Gabor response for $\theta=0^{\circ}$ (a), Gabor response for $\theta=90^{\circ}$ (b) and overall response of Gabor 12 filter bank

The 12 thresholded images resulting from the 12 Gabor filters are then combined into a single image using the logical OR operation (i.e. If one of the images has a white pixel the corresponding pixel in the aggregated image will be set to white). The aggregated image is shown in Figure 6 (a).

Figure 6 (a) detects most of the desired features detections, but the outer contour of the letter ' $S$ ' is not fully detected. To make sure that all the contours are properly closed, dilation operation is performed 8 times and then erosion is also carried out 8 times. This will close of contours while maintaining line thicknesses the same, in general. Then a hole filling operation is implemented. The resulting image, with a better contour describing ' $\mathrm{S}$ ' is shown in Figure 7 (a). In Figure 7 (a), the linear lane marking near the top right corner appears as broken, due to its small thickness. However, in reality, there exists a ' $U$ ' shaped contiguous contour starting from and finishing in the top edge of the image. Figure 7 (b) shows the image obtained by the logical OR of Images 6 (c) and (7a). For the unclosed contours that touch the image edges at two, or more, places, a 'closing' scheme is implemented so that parts of the image edge completes the contour. Then, all closed contours (i.e. blobs) are filled for the holes with the imfill function. This image is shown in Figure 7 (c). The foregoing process completes the region segmentation operation. 


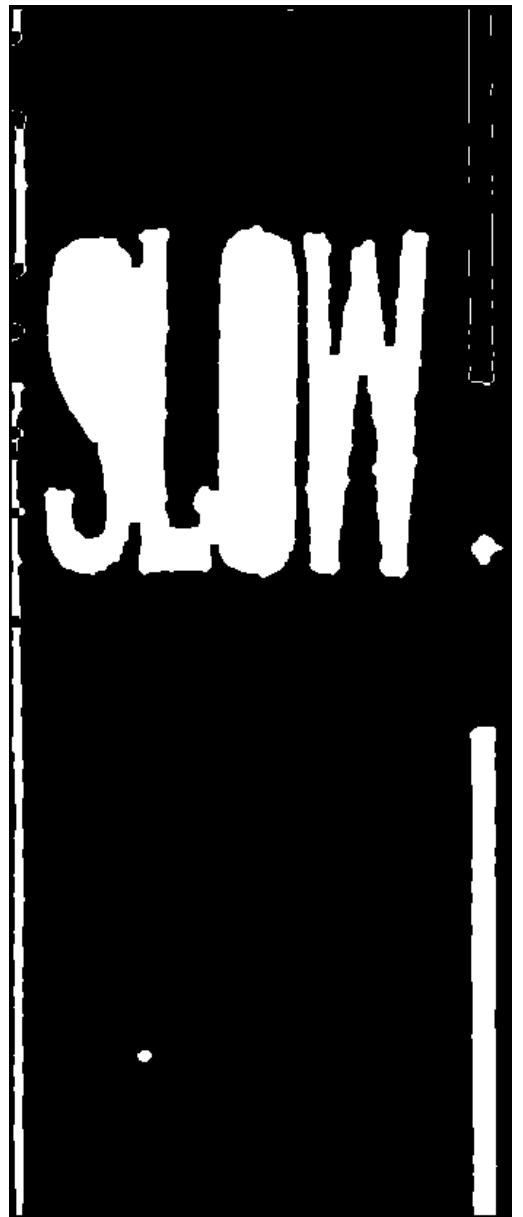

(a)

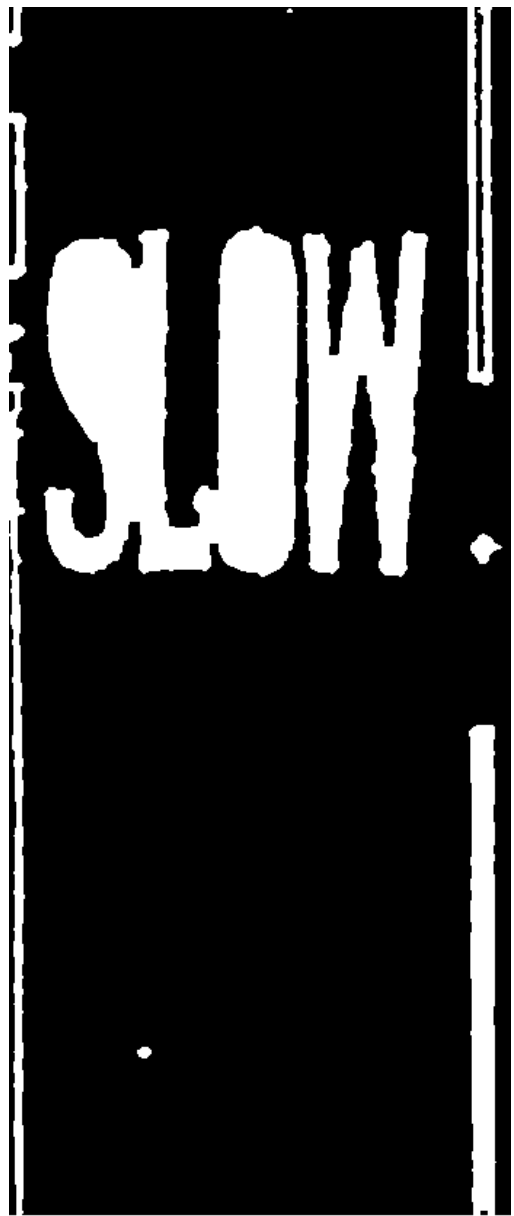

(b)

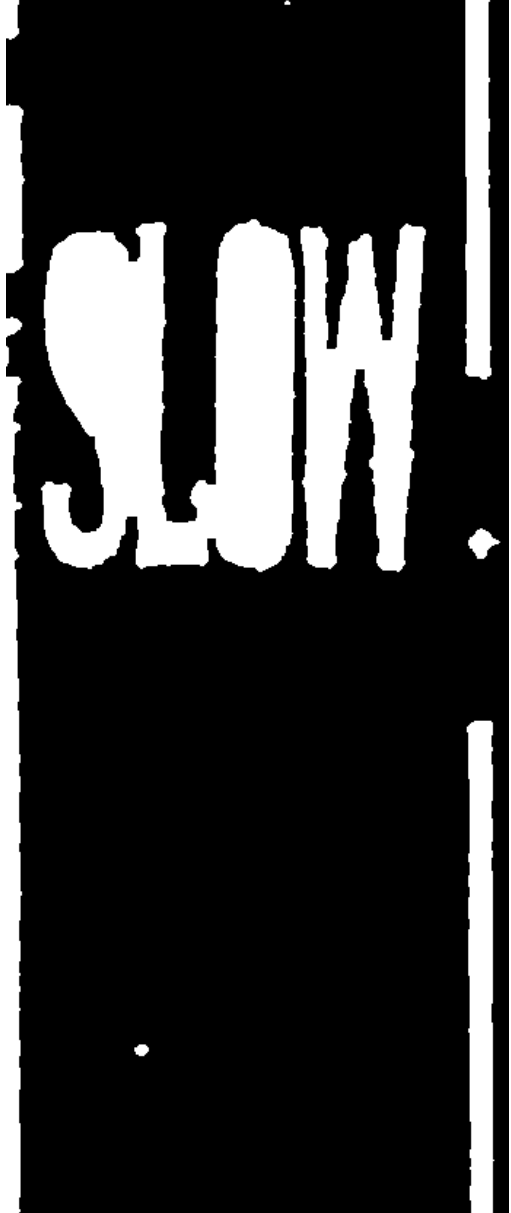

(c)

\section{RESULTS}

Figures 8 (a) and 8 (b) show the detected boundaries embedded in original intensity and range images, respectively. The sand patch test covers an area of $250 \times 250 \mathrm{~mm}^{2}$, in general [20]. This amounts to an a square area of $62 \times 62$ pixels in the image as 1 pixel is $4 \times 4 \mathrm{~mm}^{2}$. Figure 8 (c) highlights the square tiles that are on the road surface and will be analyzed for raveling.

The raveling is proposed to be quantified by the amount of range variation found within a window of $62 \times 2$ pixels. The measure of standard deviation of the range values inside a window is used here. However, if the standard deviation, within a window, alone is considered as a measure of raveling, it may lead to erroneous results. For example, within the window highlighted in red in Figure 8 (c), the surface profile of the road changes drastically. 


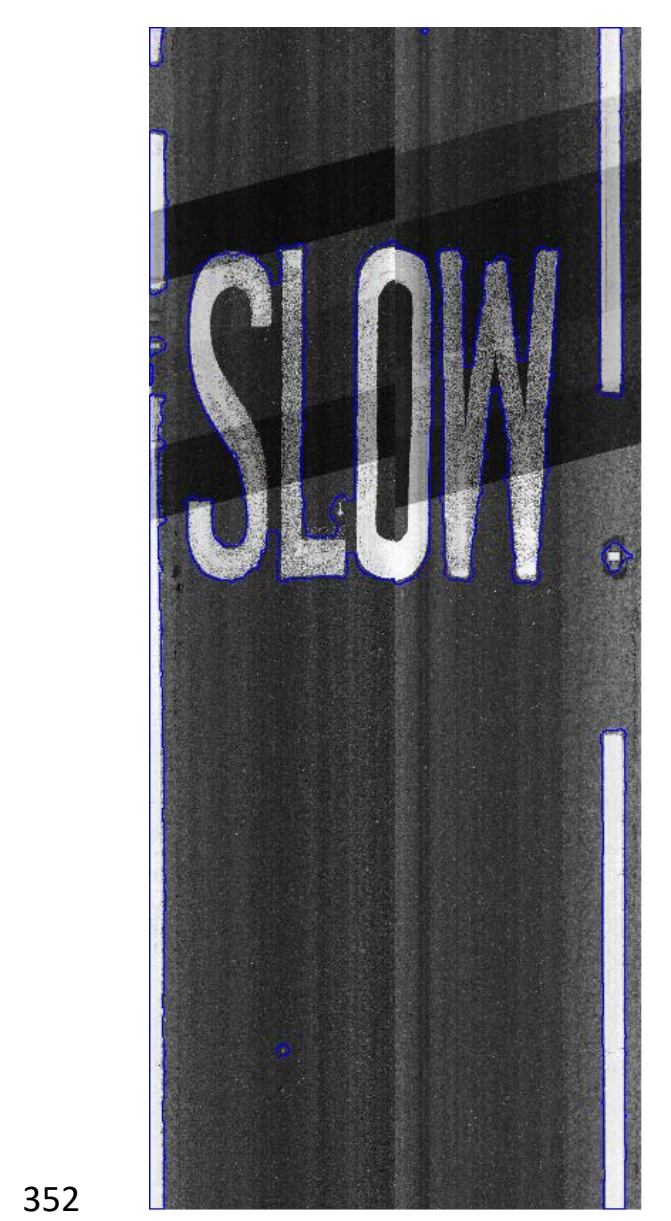

353

354

355

356

357

358

359

360

361

362

363

364

365

(a) standard deviation $(\sigma)$ of 3.9 .

$g_{i}(x, y)=A e^{-\frac{1}{2 \sigma^{2}}\left[\mathrm{x}^{2}+y^{2}\right]}$

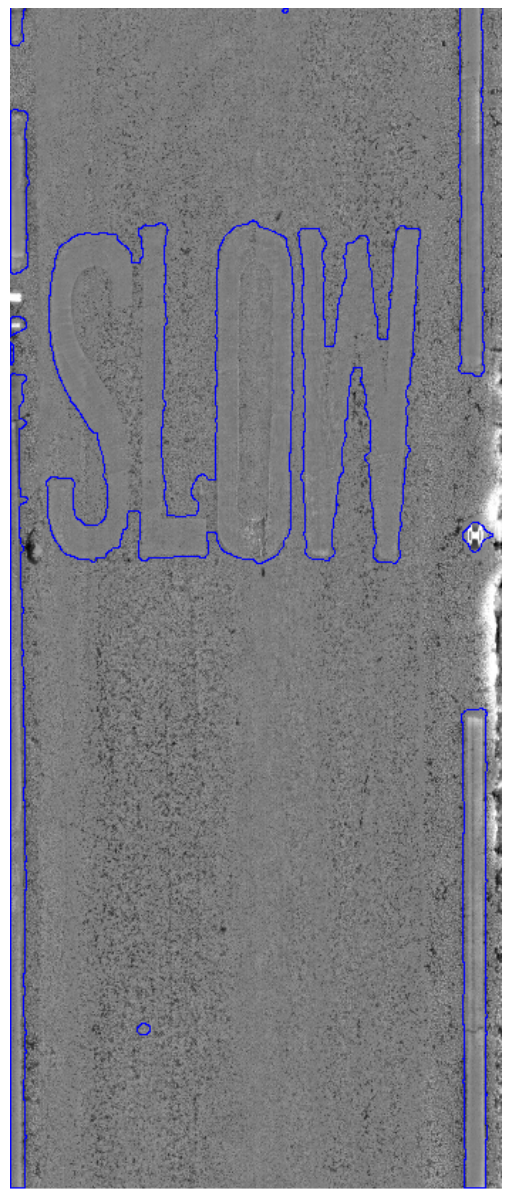

(b)

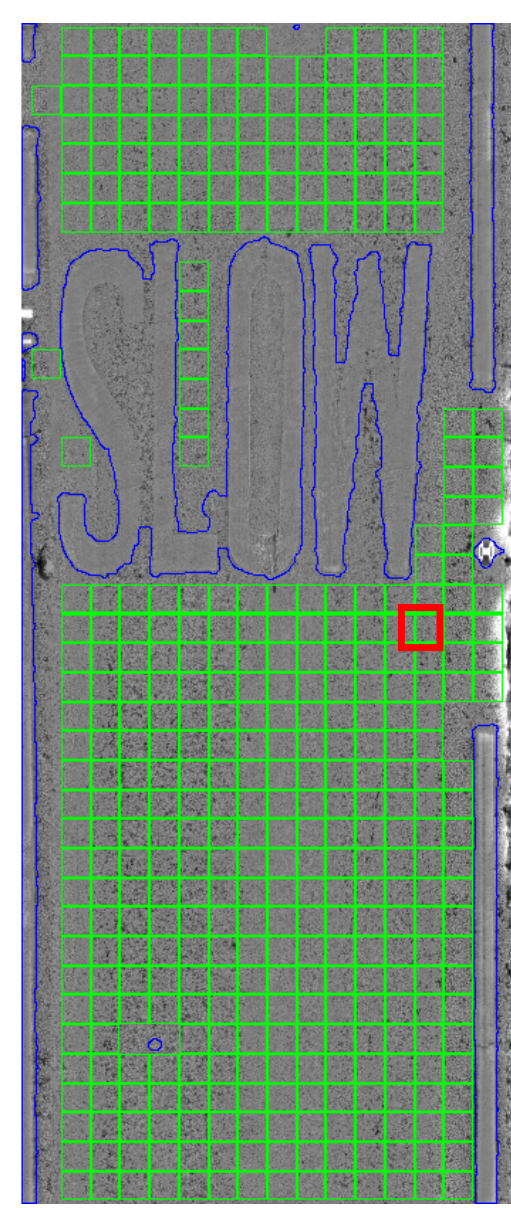

(c)

FIGURE 8 Detection embedded in the original intensity (a) and range (b) images, and the tiles to be analyzed for raveling (c).

The range data for this window is plotted in 3D in Figure 9(a) and it can be seen that the road profile is a low frequency variation. Hence, only the high frequency variations of the road must be considered for raveling detection. Here, 3D window data are proposed to be filtered with a Gaussian filter mask of 5 x 5 size with a

A Gaussian is given by the following function with $A$ being a constant ,

The designed Gaussian mask is $0.0455\left[\begin{array}{lllll}0.768 & 0.848 & 0.876 & 0.848 & 0.768 \\ 0.848 & 0.936 & 0.968 & 0.936 & 0.848 \\ 0.876 & 0.968 & 1.000 & 0.968 & 0.876 \\ 0.848 & 0.936 & 0.968 & 0.936 & 0.848 \\ 0.768 & 0.848 & 0.876 & 0.848 & 0.768\end{array}\right]$. This is a normalized mask.

The 3D data of the window, when convoluted with this mask, results in the low-pass data shown in Figure 9

(b). In Figure 9 (b) all high frequency components in the original data are removed. Now, the low-pass data, in

Figure (9b), is subtracted from the original data in Figure 9 (a) to reveal the high frequency changes to which 370 raveling contributes. This is high-pass filtered data in effect. The high frequency variations are shown in Figure 9 (c). 
(a)

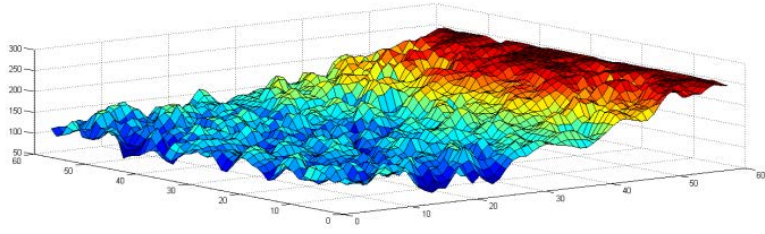

(b)

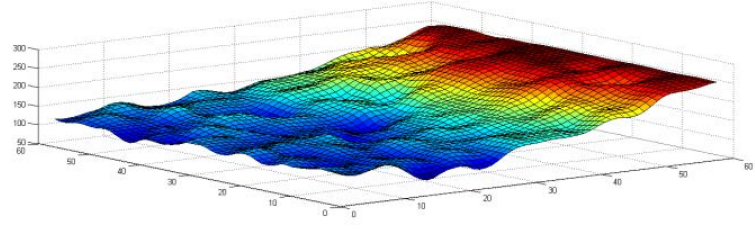

(c)

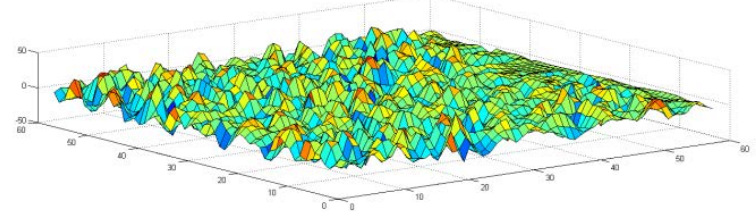

FIGURE 9 The range data of a 62 x 62 pixel window (a), its low-pass filtered form (b) and its high-pass filtered form(c)

The standard deviation of the high-pass filtered data is 11.66 given in the 8 bit range [0 255], as the range image is 8 bits, called units hereafter. If the conversion factor between the range image data and the physical range value, in meters, the standard deviation value can also be expressed in meters. In this paper, the standard deviation value above is used to quantify the amount of raveling in that particular window.

The above algorithm was tested on the 900 2D-3D image pairs, and the maxim and minimum value of standard deviation of the high-pass filtered window data, among all range images, are 17.4 and 2.9 units, respectively. The raveling condition for a window, thus found, is proposed to be classified as good, average or bad, denoted by the window highlighted in green, orange or red colour. Here, windows with standard devition less than 5 unit are characterized as good, the ones that fall in the range of $5-10$ units are branded average and the windows that have standard devitions greater than 10 units are considered as badly raveled. Figures 10 and 11 show two intensity-range image pairs with the third images showing the range image showing the detections. 


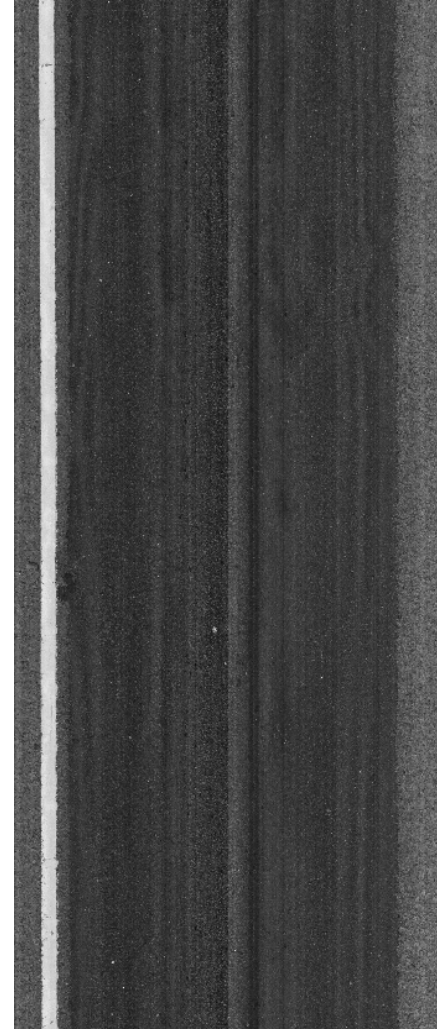

389

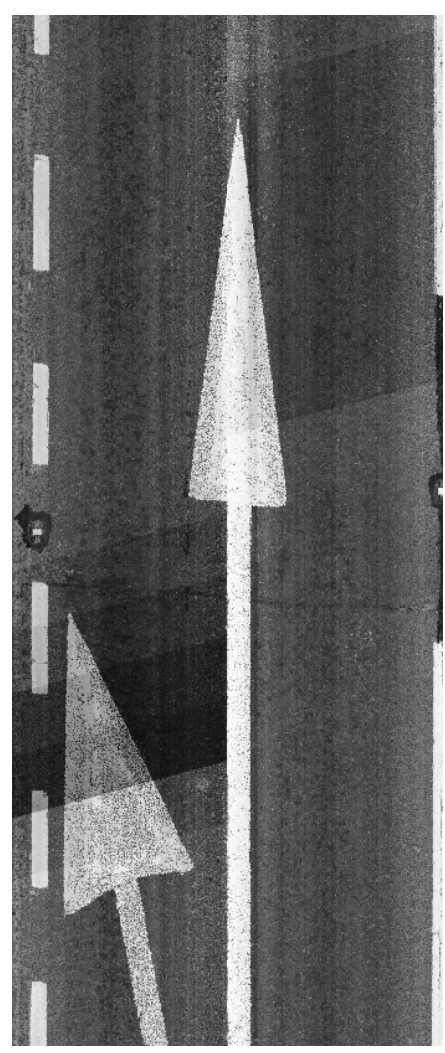

(a)

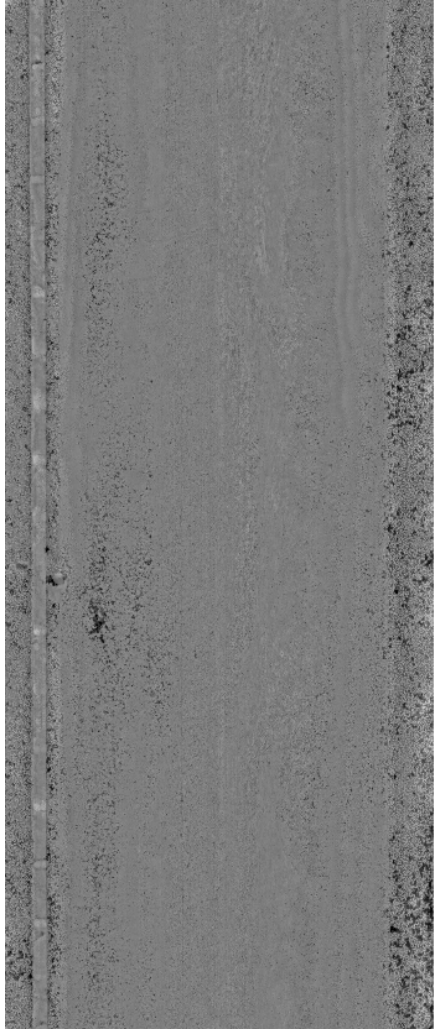

(b)

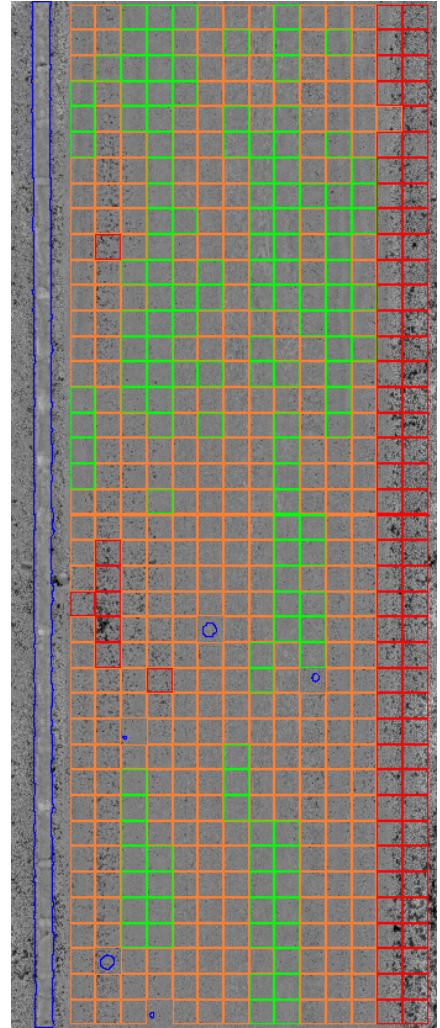

(c)

FIGURE 10 Intensity(a), range(b) and detection embedded range (c) images

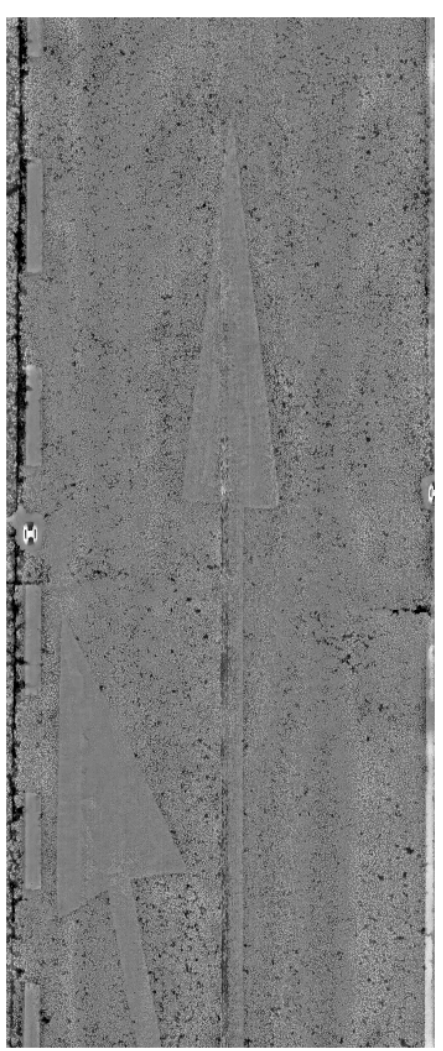

(b)

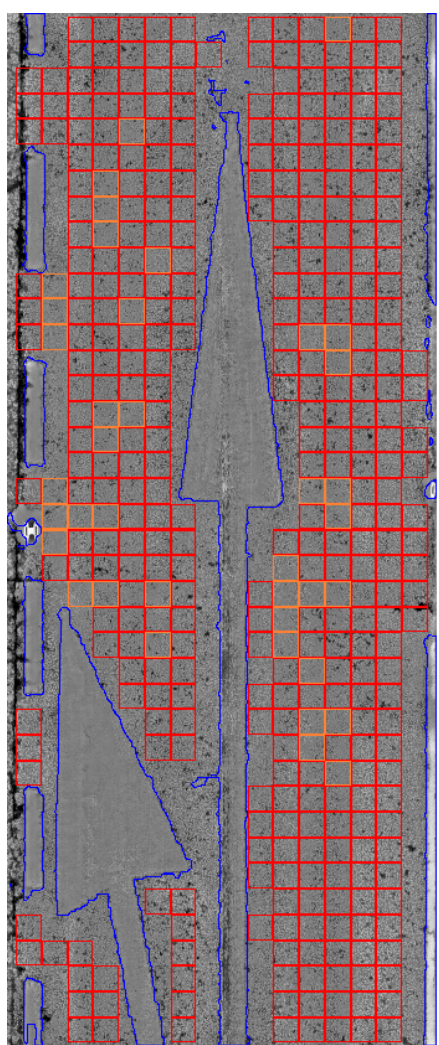

(c)

FIGURE 11 Intensity(a), range(b) and detection embedded range (c) images 
In the supplied image set of 900 pairs, the road surface is correctly detected in all images. Very rarely, small islands of road surface is is characterized as non-road, e.g. the ones surrounded by blue contours immediately above and to the left of the longer arrow in Figure 11(c). This usually happens when the local intensity of the road surface, in the 2D image, is at its highest. However, when considering all 900 images these false negatives are found to be extremely rare $(<<1 \%)$.

The range images supplied by Dynatest are preprocessed with proprietary software that is available with the hardware. Hence, the actual depth values are not valuable for the range images. If a conversion factor is available to translate the range image intensities to depth values in meters, the raveling can be expressed in either volumetric format (i.e. cubic meters per a $250 \times 250 \mathrm{~mm}^{2}$ window) or as a roughness-like value in meters. Furthermore, in the presence of a conversion factor, a bench marking process can be devised so that the raveling measure proposed here can be correlated to some standard procedures to detect raveling, e.g visual survey.

\section{CONCLUSIONS}

This paper provides a methodology, for the first time, to detect and quantify raveling from 2D and 3D images that are captured in a synchronous manner. Using an array of methods available within image processing, it has been shown that road surfaces can be accurately segmented from other painted areas on the road. Additionally, signal processing methods are used to process and measure raveling from the 3D range images. 
1. Mathavan, S., M Rahman, and K., Kamal. Application of texture analysis and the Kohonen map for the region segmentation of pavement images for crack detection, No 2304, In Transportation Research Record: Journal of the Transportation Research Board, Transportation Research Board of the National Academies, Washington, D.C., 2012, pp. 150-157

2. Bursanescu, L., M. Bursanescu, M. Hamdi, A. Lardigue, and D. Paiement. Threedimensional infrared laser vision system for road surface features analysis, Proc SPIE, Vol. 4430, 2001, pp.801-808 801-808.

3. Si-Jie, Y., S. Sukumar. 3D reconstruction of road surfaces using an integrated multi-sensory approach. Opt. Lasers Eng. 45(7), 2007, pp. 808-818.

4. Ahmed, M.F.M. and C.T. Haas. The Potential of Low Cost Close Range Photogrammetry towards Unified Automatic Pavement Distress Surveying. CD Rom. Transportation Research Board of the National, Washington, D.C., 2010.

5. Jong-Suk Y, M Sagong, J.S. Lee. Feature extraction concrete tunnel liner from 3D laser scanning data, NDT\&E International, 2009, pp. 97-105

6. Wang, K.C.P. and G. Weiguo. Automated Real-Time Pavement Crack Detection and Classification, Final Report, NCHRP IDEA 20-30/IDEA 111, Burtch, Robert, LIDAR Principles and Applications, 2002 IMAGIN Conference, Traverse city, MI, 2002.

7. Wang, K.C.P. Automated survey of pavement distress based on 2D and 3D laser images, MBTC DOT 3023, 2011.

8. Laurent J., D. Lefebvre., E. Samson. Development of a New 3D Transverse Laser Profiling System for the Automatic Measurement of Road Cracks, Proc. 6th Int. Symp. on Pavement Surface, 2008

9. Wang, K.C.P., Hou, Z, and Williams, S. Precision Test of Cracking Surveys with the Automated Distress Analyzer, ASCE Journal of Transportation Engineering, 2010

10. Q Li, Y.Ming, Y. Xun, B. Xu. A real-time 3D scanning system for pavement distortion inspection.. Measurement Science and Technology, vol. 21 issue 1 January 01, 2010. p. 015702-015702.

11. Sun X, J. Huang, W. Liu, and M Xu. Pavement crack characteristic detection based on sparse representation, EURASIP, Journal on Advances in Signal Processing, 2012, pp. 191

12. FHWA, U.S. Department of Transportation. Long-term Pavement Performance (LTPP). Standard Data Release 26.0 DVD, 2012.

13. Kringos, N., A.Scarpas. Raveling of Asphaltic Mixes Due to Water Damage: Computational Identification of Controlling Parameters, In Transportation Research Record: Journal of the Transportation Research Board, No 1929, Transportation Research Board of the National Academies, Washington, D.C., 2005.

14. Tuceryan, M and A.K.Jain. Texture Analysis, In The Handbook of Pattern Recognition and Computer Vision (2nd Edition), by C. H. Chen, L. F. Pau, P. S. P. Wang (eds.), World Scientific Publishing Co., 1998, pp. 207-248.

15. Lemaitre, G. and M. Rodojevic. Texture segmentation: Co-occurrence matrix and law's texture mask methods, Technical report, 2010. Available at http://g.lemaitre58.free.fr/pdf/vibot/scene_segmentation_interpretation/cooccurencelaw.pdfXXXXXX 
16. Helli, B., and M.E.Moghaddam. A text-independent Persian Writer Identification based on Feature Relation Graph (FRG), Pattern Recognition 43, 2010, pp 2199-2209

17. Salman, M., S.Mathavan, K.Kamal, and M.Rahman. Pavement Crack Detection Using the Gabor Filter, 16th International IEEE Annual Conference on Intelligent Transportation Systems, October 6-9, 2013, The Hague, The Netherlands.

18. what-when-how 2013, Accessed on $26^{\text {th }}$ July, 2013 from http://what-when-how.com/introduction-to-videoand-image-processing/morphology-introduction-to-video-and-image-processing-part-1/

19. MATLAB, Accessed on $6^{\text {th }}$ July 2013 from http://www.mathworks.co.uk/help/images/ref/bwmorph.html

20. BS EN 13036-1: Road and airfield surface characteristics - Test methods - Measurement of pavement surface macrotexture using a volumetric patch technique, BSI. 